\title{
INTELIGÊNCIA ARTIFICIAL: UMA ANÁLISE DOS IMPACTOS E DOS POTENCIAIS DAS INTELIGÊNCIAS ARTIFICIAIS NA SEGURANÇA DOS ESTADOS
}

\section{ARTIGO ORIGINAL}

SGROTT, André Luiz Bezerra ${ }^{1}$

GONZALEZ, Rodrigo Milindre ${ }^{2}$

SGROTT, André Luiz Bezerra. GONZALEZ, Rodrigo Milindre. Inteligência Artificial: Uma análise dos impactos e dos potenciais das Inteligências Artificiais na segurança dos Estados. Revista Científica Multidisciplinar Núcleo do Conhecimento. Ano 06, Ed. 01, Vol. 03, pp. 61-86. Janeiro de 2021. ISSN: 2448-0959, Link de acesso: https://www.nucleodoconhecimento.com.br/tecnologia/seguranca-dosestados

\section{RESUMO}

O advento de tecnologias futurísticas transforma a ótica pela qual vemos o mundo e o que acontece nele. As Inteligências Artificiais surgem com um papel incerto nas Relações Internacionais, a integridade de suas capacidades e potenciais ainda estão por serem descobertas, mas em diversas tarefas elas já superam os seres humanos, provando que poderão se tornar ferramentas decisivas para o futuro dos Estados e da civilização humana. Neste sentido, o objetivo deste artigo é identificar de que forma o uso da Inteligência Artificial na função de ferramenta estatal, como ela impactará a balança de poder global e segurança dos Estados. Pesquisa foi conduzida pelo método dedutivo e qualitativo, pautado na pesquisa bibliográfica e documental.

\footnotetext{
${ }^{1}$ Graduação em Relações Internacionais.

2 Orientador. Doutorado em andamento em Estudos Estratégicos Internacionais. Mestrado em Estudos Estratégicos Internacionais. Graduação em Relações Internacionais.
} 
Utilizando a ótica neorrealista, vemos que as nações do mundo entraram em uma corrida armamentista tecnológica focada em desenvolver sistemas de inteligência artificial que possam dar a elas séria vantagem, contra seus competidores nos setores econômicos, militar e político. Durante o decorrer da pesquisa foi possível identificar que as Inteligências Artificiais terão um grande impacto nas estruturas estatais, porém, por ser uma tecnologia em sua infância, a extensão destes impactos ainda é uma incógnita. Assim o recente aumento do interesse público por essas tecnologias traz à luz questionamentos de quais serão os seus resultados e impactos nas vidas dos cidadãos, porém, seja mais pertinente questionarmos quais serão os impactos dela nos Estados.

Palavras-chave: Inteligências Artificiais, segurança, cibernética, tecnologia, neorrealismo.

\section{INTRODUÇÃO}

No início de setembro de 2017, o presidente russo, Vladimir Putin, trouxe a inteligência artificial dos laboratórios do Vale do Silício, da academia e do porão do Pentágono para a vanguarda da política internacional: A inteligência artificial é o futuro, não apenas para a Rússia, mas para toda a humanidade. Ela vem com oportunidades colossais, mas também ameaças que são difíceis de prever. Quem quer que se torne o líder nesta esfera se tornará o governante do mundo (RUSSIA, 2020). A introdução das Inteligências Artificiais nos círculos de debate e pesquisa das relações internacionais está se provando cada vez mais pertinente para os estudos de segurança estatal. O conceito de IA como uma ferramenta geopolítica é inovador, familiar, porem abstrato, mas os potenciais estratégicos desta tecnologia devem ser compreendidos e analisados para que possamos nos preparar para uma possível mudança da forma que política, segurança e diplomacia operam.

Assim, a pesquisa guiou-se pelo seguinte questionamento: De que forma o uso da Inteligência Artificial como ferramenta geopolítica impactará a balança de poder global e a segurança dos Estados? Para isso, o objetivo geral é Identificar de que forma o uso da Inteligência Artificial como ferramenta estatal, impactará a balança de poder 
global e a segurança dos Estados. Para alcançar uma construção completa da pesquisa é necessário, expor o conceito de Inteligência Artificial e como ela pode ser aplicada, destacar como as contribuições Teóricas nas Relações Internacionais podem explicar a interação dos Estados em competição e identificar de que forma os usos dessa tecnologia como uma ferramenta geopolítica podem interferir na Segurança dos Estados, esses são os objetivos específicos do trabalho.

Por consequência, o artigo aborda algumas as aplicações civis e militares das IAs, bem como suas definições e potenciais no âmbito de ferramenta de poder dos Estados ao supor que o sistema internacional é regido por uma ótica neorrealista das Relações Internacionais, como ela influencia a percepção dos Estados a respeito dos poderes militares clássicos dando uma nova espada para a cyber-segurança. A partir desses fatos, constrói-se uma base teórica para o assunto, que é o Impacto dos sistemas de IA como ferramenta dos Estados e uma consequente plangem do campo competitivo internacional. Em luz das informações apresentadas acima, para tal, em primeiro momento, a pesquisa foi embasada em livros e artigos acadêmicos nas áreas de tecnologia da computação e IAs e de Relações Internacionais tanto neorrealistas, quanto Cibernéticas[3], onde se extraem abstrações por vezes futurológicas[4]. Pesquisa foi conduzida pelo método dedutivo e qualitativo, pautado na pesquisa bibliográfica e documental.

A primeira seção é focada em explorar e explicar o que é uma Inteligência Artificial, os processos que envolvem suas funções e sua aplicabilidade nas áreas civis e militares. Assim, por ser uma seção técnica ela é dívida em dois tópicos: o introdutório elabora nas definições de IA e suas funções de raciocínio, pois é vital que compreendamos o que é esta ferramenta e como ela opera, para que possamos elaborar no seu papel dentro do Estado; o segundo que trata das aplicabilidades das IAs, nas áreas militares e civis, como uma ferramenta de alta tecnologia capaz de maximizar as funções que forem designadas a ela. O seu principal objetivo, além de elaborar as definições e aplicabilidades dos sistemas de IA é expandir a compreensão dos potenciais da tecnologia, para que as próximas seções dialoguem com o assunto. 
A segunda seção aborda as bases teóricas das Relações Internacionais que darão sustância a seção seguinte. A seção aborda a teoria neorrealista clássica estrutural de Waltz e ofensiva de Mearsheimer, uma vez que o tema dá centralidade aos Estados é apresentada uma crítica ao instrumentalismo tecnológico dessas teorias por não experimentarem a perspectiva digital, as quais partem de premissas com viés industrialista cujo objetivo é a influência das IAs nos Estados e, consequentemente, nas relações internacionais.

A terceira seção expõe as influências dessa tecnologia na balança de poder e na segurança dos Estados, levantando exemplos históricos do uso de tecnologias inovadoras que moldaram o rumo da história em favor dos seus usuários. Nesta seção também é abordada a utilidade e fragilidades dos sistemas clássicos de segurança, em vista de uma tecnologia magnificação de poder como as IAs e IAGs[5], e como ela pode nivelar os poderes de atores nacionais e atores não estatais no sistema internacional.

Assim, é importante que pesquisas desta envergadura sejam realizadas nas Relações Internacionais, pois o incremento da tecnologia no campo da segurança cria uma outra perspectiva sobre o assunto, trazendo muitas incertezas e novas ameaças para a área. Logo, é intrínseco que busquemos nos atualizar com os tempos atuais e nos preparar para as possíveis mudanças no cenário internacional.

\section{INTELIGENCIA ARTIFICIAL}

Nesta seção abordar-se-ão as definições do que é uma inteligência artificial e seus potenciais aplicabilidades. Primeiramente, será abordado as definições da tecnologia, levantando-se uma série de definições para que possamos abordar, em seguida, suas aplicabilidades. Por segundo será tratado das aplicabilidades dessa tecnologia em diferentes áreas de operação, baseando-se em artigos técnicos e análises estratégicas de diferentes órgãos de segurança. 


\subsection{O QUE É UMA INTELIGÊNCIA ARTIFICIAL?}

A compreensão do que é uma Inteligência Artificial foi levantada como o elemento central desta seção, pois entendê-la é fundamental para a compreensão do restante da pesquisa. A definição moderna de Inteligência Artificial (ou IA) é "o estudo e o design de agentes inteligentes" (MCCHARTHY, 2007), onde um agente inteligente é um sistema que percebe seu ambiente e executa ações que maximizam suas chances de sucesso. John McCarthy (2007), que cunhou o termo em 1956, define-o como "a ciência e a engenharia de fabricação de máquinas inteligentes".

Desde 1950, a capacidade de uma máquina de agir humanamente foi avaliada com base no teste de Turing[6]. Segundo Landgrebe e Smith (2019) para passar o teste, uma máquina deve ser capaz de se envolver em um diálogo por pelo menos cinco minutos de tal forma que um ser humano (o interrogador) não consiga distinguir seu comportamento daquele de um ser humano. Os autores elaboram mais a respeito das qualificações do teste citando que para agir humanamente, uma máquina teria que atender a duas condições (I) reagir de forma adequada à variação no diálogo humano e (II) exibir uma personalidade e intenções semelhantes às humanas (LANDGREBE; SMITH, 2019).

De acordo com os autores Landgrebe e Smith (2019), nenhuma máquina passou no teste até agora, e alguns pesquisadores acreditam que, por razões matemáticas, seria realmente impossível programar uma máquina que possa dominar o padrão complexo e evolutivo de variação contidos nos diálogos humanos. Nilsson (2010) define, por exemplo, inteligência artificial como a "[...] atividade dedicada a tornar as máquinas inteligentes" enquanto a inteligência é aquela qualidade que permite a uma entidade funcionar de forma adequada e com visão de futuro em seu ambiente. Acompanhando o pensamento de Nilsson, a High-Level Group on IA da Comissão Europeia apresenta um entendimento mais compreensivo do termo:

Os sistemas de inteligência artificial (IA) são sistemas de software (e possivelmente também de hardware) projetado por humanos que, atendendo a um objetivo complexo, atuam na dimensão física ou digital percebendo seu ambiente por meio da aquisição de dados, interpretando 
os dados estruturados ou não estruturados coletados, raciocinando sobre o conhecimento ou processando as informações, derivado desses dados e decidindo a melhor ação (ões) para alcançar o objetivo determinado. (HIGH-LEVEL EXPERT GROUP ON ARTIFICIAL INTELIGENCE, 2019, p. 6, tradução nossa).[7]

Porém, existem duas abordagens principais para IA amplamente concebidas. $\mathrm{O}$ primeiro é a inteligência artificial simbólica - a criação de sistemas especialistas e regras de produção para permitir que uma máquina deduza caminhos comportamentais. Como descrevem Campbell, Hoane e Hsu (2002), o Deep Blue da IBM, que derrotou Garry Kasparov no xadrez em 1997, usou uma abordagem simbólica. Carbonell, Michalski e Mitchell (1972) afirmam que, as abordagens computacionais ou conexionistas da inteligência artificial, em contraste, tipicamente tentam permitir o reconhecimento do problema e a ação de máquinas por meio de cálculos, em vez de representação simbólica. Os autores Nguyen, Yosinski e Clune (2015) acrescentam que, o Machine Learning[8] representa uma abordagem computacional chave para a inteligência artificial. Várias técnicas computacionais são usadas para criar algoritmos de Machine Learning, incluindo redes bayesianas, árvores de decisão e Deep Learning.

Deep Learning, agora popularmente associado à inteligência artificial, é uma técnica que aproveita redes neurais para treinar algoritmos para realizar tarefas específicas, como reconhecimento de imagem (NGUYEN; YOSINSKI; CLUNE, 2015). Alguns pesquisadores estão buscando abordagens híbridas que integram partes da aproximação simbólicas e computacionais para IA. A esperança por trás das abordagens híbridas é que a criação de linguagens comuns permitirá que algoritmos que possam empregar vários caminhos para aprender como fazer tarefas específicas, tornando-os mais eficazes (LIETO; CHELLA; FRIXIONE, 2017).

Em 2019 o Departamento de Defesa dos Estados Unidos da América em uma simulação de combate, no qual um pelotão atacaria uma posição fortificada com a guarnição de defesa constituída por uma companhia. Nesta simulação seriam utilizadas Inteligências Artificiais e robôs de combate teóricos, que seriam implementados como ferramentas de suporte operacional para os soldados de apenas 
um dos lados (os atacantes), que estariam competindo contra as forças de defesa, em completa desvantagem numérica. A operação foi presidida pelo Capitão Philip Belanger do exército dos EUA, realizada pelo laboratório de manobras de batalha da base militar estadunidense Fort Benning. A missão constituía-se de:

Desalojar uma companhia de infantaria de defesa, cerca de 120 soldados, com um único pelotão de apenas 40 atacantes a pé. Essa é uma tarefa que normalmente seria atribuída a um batalhão de mais de 600. Em outras palavras, em vez da superioridade mínima de 3: 1 em números que a tradição militar requer para um ataque bem-sucedido, a força simulada de Belanger estava em menor número 1: 3. (FREEDBERG. 2019. N.P. tradução nossa).[9]

Freedberg (2019) elaborou que, os resultados do teste se provaram extremamente positivos para a companhia municiada com as "tecnologias futurísticas", reduzindo os riscos de casualidades e fatalidades do engajamento para quase zero. Freedberg (2019) elabora nas palavras de Ted Mancubia dizendo, este teste foi realizado com tecnologias que ainda não existem, mas são ferramentas que a indústria bélica e os laboratórios do exército poderiam criar. Isto é apenas uma prova teórica das capacidades máximas desta ferramenta, na segunda parte deste capito abordaremos com mais detalhes suas aplicações.

\subsection{APLICAÇÕES DA INTELIGÊNCIA ARTIFICIAL}

Como destacado anteriormente, as IAs estão se tornando tão capazes quanto os seres humanos na realização de algumas tarefas específicas. Como toda ferramenta, a Inteligência Artificial tem vários usos e aplicações, sendo ela uma um operador virtual, pode ser aplicada em diversas áreas que comportem um modelo de atuação digital. É difícil de apontar todas as capacidades e o potencial de um aparato tão jovem, mas utilizando os estudos realizados por grupos de pesquisa de vários polos tecnológicos voltados para o aprimoramento das técnicas operacionais das IAs, mesmo em sua infância podemos citar suas possíveis áreas de aplicação. Para isso é essencial explicar as quatro principais áreas de aplicabilidades da IA em um cenário pratico: Reconhecimento de Imagens; Análise de Textos; Veículos de Pilotagem Autônoma; e Game-Playing será abordado, cada uma, individualmente. 
Uma observação conhecida como paradoxo de Moravec destaca como tarefas que são fáceis para as pessoas geralmente são difíceis para os computadores e vice-versa (MORAVEC, 2015). Segundo Morgan, et al. (2020), um exemplo desse paradoxo é a imagem reconhecimento, os humanos reconhecem as imagens facilmente $e$ inconscientemente, mas por muitos anos foi um desafio muito além da capacidade dos computadores.

De acordo com o artigo técnico de He et al. (2015), recentemente devido ao progresso principalmente em redes neurais profundas, a visão computacional experimentou uma etapa de transição no reconhecimento de imagem e detecção de objetos e agora excede a habilidade humana em algumas dessas tarefas, embora ainda possa exibir falhas surpreendentes em comparação com os humanos. Os avanços nesta área também foram impulsionados pela proliferação de imagens e, principalmente, imagens rotuladas. Agora disponíveis, redes neurais profundas são "treinadas" ao serem expostas a altos números de imagens rotuladas. O progresso se estende ao reconhecimento facial e aos mais sutis aspectos da expressão facial, tornando a tecnologia de identificação biométrica através do rosto, um recurso que torna até mesmo análise emocional, algo viável. Do ponto de vista militar, a capacidade de detectar objetos e reconhecer imagens e, principalmente, para reconhecer rostos e realizar análises emocionais, tem aplicações claras.

Observando o progresso recente de redes neurais profundas às grandes quantidades de dados digitais agora disponíveis, também resultaram em avanços rápidos em vários aspectos da natureza do processamento de linguagem. De acordo com Le e Schuster (2016), embora o desempenho de nível humano em tarefas pesadas de linguagem esteja se provando ser mais evasivo do que era para as imagens, a tradução automática tornou-se uma opção viável em um número crescente de aplicações e contextos. Também houve avanços substanciais em aplicativos como resumo e análise de sentimento, para não mencionar os motores de busca, embora muito do progresso nesses campos tenha sido feito usando abordagens diferentes das redes neurais (GAMBHIR; GUPTA, 2016). 
Em 2004, quando a DARPA[10] sediou seu primeiro Grande Desafio, os veículos sem motorista foram encarregados de atravessar 142 milhas no deserto, o mais longe que qualquer um deles viajou antes de falhar foi apenas 7,5 milhas, e nenhum competidor foi declarado como vencedor. Hoje, os veículos autônomos estão sendo testados nas estradas em várias cidades ao redor do mundo, estes veículos ainda são controversos e envolveram-se em vários acidentes fatais, mas começam a ganhar a confiança dos motoristas e passageiros (MORGAN et al., 2020).

Atualmente, esses sistemas exigem que humanos estejam disponíveis para intervir da mesma forma que pilotos são exigidos quando a aeronave está no piloto automático; usando a terminologia de sistemas de armas, referem-se a eles como "sistemas autônomos supervisionados" (MORGAN et al., 2020). Ainda há intenso debate público sobre a segurança desses sistemas. A evolução do debate pode ser reveladora, tanto sobre a eficácia da supervisão como um mecanismo de segurança e de que forma a culpa pode ser atribuída em acidentes envolvendo autonomia supervisionada.

De acordo com Metz (2016a), outro ponto de referência comum para medir o progresso em IA ao longo de sua história tem sido a capacidade de jogar. Quando o Deep Blue da IBM venceu Gary Kasparov no xadrez em 1997, o evento foi considerado um grande feito em IA. Um evento igualmente importante ocorreu quando AlphaGo do Google DeepMind venceu Lee Sedol em Go em 2016.

Em termos da complexidade das posições do tabuleiro e do número de movimentos possíveis, Go é um jogo muito mais difícil do que xadrez, então entusiastas de Go, e até pesquisadores de IA, pensaram que seria mais uma década antes que os computadores alcançassem um desempenho sobre-humano nesse jogo (METZ, 2016b, não paginado, tradução nossa).[11]

Em outros aspectos, entretanto, xadrez e Go são jogos relativamente simples, as regras são claras, os movimentos são sequenciais, e ambos os jogadores têm informações completas sobre o estado dos jogos. Um desafio maior é apresentado em jogos como o pôquer, onde os jogadores têm apenas informações e blefes estão envolvidos. Esses jogos estão fora do alcance dos computadores para muitos anos, 
mas os sistemas de IA recentemente derrotaram os jogadores profissionais de pôquer (RILEY, 2016). Segundo Kim e Lee (2017), esforço está em andamento para desenvolver IA que possa jogar jogos como StarCraft, os quais envolvem várias camadas de ação estratégica em uma linha do tempo estendida com muito mais movimentos do que o típico jogo Go ou mão de pôquer.

Esses sistemas de IA para jogos tendem a se basear em uma abordagem chamada aprendizagem por reforço, que não é exatamente nova, mas vem ganhando popularidade rapidamente. Considerando que para o reconhecimento de imagem, normalmente, é necessário um grande conjunto de imagens e suas classificações associadas, para aprendizagem por reforço, o que é necessário é atribuir pontuações ou "recompensas" ao "agente" de IA enquanto ele toma decisões, fazendo a transição de seu ambiente de um estado para outro. Segundo Levine et al. (2017), o objetivo do agente IA é acumular as maiores recompensas possíveis, esta técnica também pode ser aplicada fora de jogar para, por exemplo, o movimento do robô. Mas, apesar desses avanços, a aplicação de IA de jogo para abstrair estratégias militares ou jogos de guerra ainda são muito ambiciosas.

Por outro lado, os benefícios das IAs na defesa nacional não são óbvios em primeira instância, mas com o uso da criatividade e algumas entrevistas com especialistas da área militar e computacional, realizadas por pesquisadores da Rand Corporation é possível identificar os principais benefícios dessa tecnologia na área militar, como a velocidade de tomada de decisões, o uso da Big Data, suporte em tomada de decisão, melhoramento na cyber defesa e trabalho e redução de custo.

Segundo a pesquisa, a categoria mais mencionada foi a velocidade da tomada de decisão. É claro que a velocidade com que uma pessoa consegue tomar uma decisão sempre será uma vantagem, seja no campo de batalha ou até em meio a câmara do congresso.

Com a ideia de que se fosse possível operar através do OODA Loop[12] mais rápido do que seus adversários, então eles serão incapazes de realizar as contra-ações necessárias para se defender contra os próprios ataques ou para gerar sua própria ofensiva opções rápidas o suficiente 
para superar as contra-ações. (MORGAN et al., 2020, p. 16, tradução nossa).[13]

Os autores acrescentam que certamente há casos em que esse tipo de vantagem pode ser imaginado, no entanto, também é importante ter em mente que os prazos nem sempre são dominados pelos processos de decisão que a IA pode ajudar a acelerar. É importante não exagerar o valor de acelerar o processo de decisão nesses casos (MORGAN et al., 2020).

A própria natureza da Big Data torna seu uso em situações que possuem um prazo curto, imprático, se não impossível. Com a ajuda de IAs, o uso da Big Data "on the fly" pode se tornar possível criando uma vantagem massiva contra o adversário. Segundo Morgan et al. (2020), dado o volume cada vez maior de dados disponíveis no mundo hoje, espera-se que as IAs continuem a aumentar em proeminência. Os próprios sistemas serão munidos de uma gama titânica de informação, podendo tomar ações de uma forma muito mais informada e variada que um humano, desprovido dessa variada informação.

O termo Big Data é geralmente usado para descrever dados que são muito grandes para serem armazenados na memória do computador, são gerados muito rapidamente para serem gerenciados por um único computador, ou muitos formulários ou formatos diferentes. Por causa desses três problemas, pode ser um desafio para os humanos dar sentido às informações contidas nos dados, mas as máquinas e a IA tendem a melhor desempenho quanto mais dados forem disponibilizados para eles. o grande volume de as informações coletadas por vários sensores são mais do que um ser humano ou equipe de humanos pode analisar (MORGAN et al., 2020, p. 17, tradução nossa).[14]

Na categoria de suporte na tomada de decisão, segundo Morgan et al. (2020), é antecipado que as IAs poderão ser capazes de recomendar opções aos tomadores de decisão de forma mais rápida. Hoje os tomadores de decisão, geralmente, possuem uma equipe de conselheiros e técnicos que fazem análises e recomendações de quais são as melhores opções para dada situação. Levando em consideração o que foi exposto acima sobre Big Data, Morgan et al. (2020) supõe que se adicionarmos uma ferramenta como a IA na equação, as recomendações, além de 
serem apresentadas de forma mais rápida, elas seriam superiores as de que um humano poderia oferecer. "Mais ambiciosamente, alguns acreditam que o progresso em jogos como Go e StarCraft indica potencial para aplicação de IA em tarefas de planejamento estratégico [...]" Morgan et al. (2020, p. 18) adiciona, "Mesmo que essas tecnologias não sejam apropriadas para uso na tomada de decisões ou sugestões de combate, os especialistas antecipam que poderiam ser usados para fornecer uma gama mais ampla de possíveis ações do adversário em jogos de guerra [...]". Não podemos deixar de considerar que as adversidades encontradas na realidade são muito mais complexas e imprevisíveis do que as encontradas no cenário virtual.

Com o acelerado avanço tecnológico, em específico à área digital, e a sua implementação nos ramos militar e civil, a guerra cibernética e o ciber-terrorismo vêm se tornando uma das principais pautas de discussão nos círculos de especialistas em defesa. Segundo Morgan et al. (2020), a forma atual de detecção de malware já não estão sendo suficientes para deter alguns programas, pois conforme as tecnologias evoluem esses "vírus" evolvem para sobrepassar as atuais barreiras estáticas. Morgan et al. (2020) adiciona, que o DARPA Cyber Grand Challange ilustrou um grande aumento do interesse no potencial de máquinas que possam encontrar e arrumar vulnerabilidades em sistemas aliados, ou achar e atacar as vulnerabilidades de sistemas adversários, mas que as elas ainda não conseguem atingir o nível de performance de um humano experiente.

[...] não é mais suficiente simplesmente usar tags estáticas para identificar malware, porque os invasores descobriram maneiras de gerar malware com menos dessas marcas. Em resposta, as empresas de antivírus analisaram seus grandes conjuntos de dados de comportamento de malware para criar IA que pode observar o software em um sistema e sinalizar ações que são identificadas como suspeitas [...] (MORGAN et al., 2020, p. 19, tradução nossa).[15]

Como se observa em todas as áreas da economia, tarefas que antes exigiam dedicação de uma ou mais pessoas, progressivamente estão sendo realizadas por IAs ou robôs. De acordo com o Morgan et al (2020, p. 19) "a Al demonstrou a capacidade de melhorar ou otimizar processos de muitos diferentes tipos, o que, por sua vez, leva a reduções de custos." Pode-se observar que esse potencial vai além 
de uma única área, pois se esta ferramenta for fundida com outras tecnologias inovadoras, como veículos auto pilotados, impressoras 3D e robôs de fabricação, podemos ver ao horizonte não tão distante uma possível nova revolução industrial.

\section{TEORIA DAS RELAÇÕES INTERNACIONAIS, PODER E TECNOLOGIA}

Waltz, pensador neorrealista e autor de Man, the State, and War (1959) e de Theory of International Politics (1979), utiliza o conceito de estado de natureza de Hobbes como principal para formatar sua teoria de Relações Internacionais e compreender a relação entre Estados dispostos em um sistema anárquico. Além disso, utiliza como base a conceituação de ordem internacional dos teóricos do realismo clássico para dar corpo à sua concepção de estrutura.

Pela falta de um poder global que os ordene, Waltz (1959) elabora que os Estados são livres para declarar guerras e fazer paz com os outros estados quando quiserem, já que se encontram em um Estado constante de natureza, a gênese que os homens se comportavam antes do surgimento do Estado. A falta de um poder regulador, apesar de oferecer uma grande liberdade, causa constante tensão, paranoia e um sentimento de insegurança e, por isso se armar e obter quaisquer vantagens sobre seus vizinhos thes confere uma chance de sobreviver e lutar contra seus competidores. Ao inferir essa definição, portanto, a guerra é inevitável, pois não há uma força maior que impeça os Estados de guerrear e garantir suas propriedades e sobrevivência. A estrutura do mundo, consequentemente, consiste em três imagens: o homem, ou a primeira imagem, (as estruturas do Estado e suas posses), a segunda imagem, o Estado (as capacidades relativas dos Estados) e, a terceira, o sistema internacional (as interações estatais na anarquia).

Waltz (1959) aborda mais claramente na sua tese as vontades do Estado, o que ele almeja e como se comporta no sistema. A irracionalidade social conduz a um processo de instabilidade local, que pode derivar no conflito. O autor elenca que as instabilidades estatais são fatores condicionantes para um conflito, já que estes podem ser objetos de justificativa para a presença militar numa determinada região, 
seja no espaço em questão ou nos territórios limítrofes deste. Ao aliar a terceira imagem do Waltz, não há mecanismos legais que impeçam a atuação de potências em Estados instáveis, e estas linhas ganham força, quando existem riquezas envolvidas nos espaços territoriais.

Waltz (1979) na sua obra The Theory of International Politics, desenvolvida no seio da Guerra Fria, pontuava que os sistemas bipolares tendem a uma maior estabilidade global na balança de poder, neste aspecto, ocorre uma maior cooperação entre as nações de cada lado com suas respectivas ideologias, alinhadas com a dos grandes centros, neste caso, claramente EUA e URSS. Esta cooperação conduz a manutenção do status quo, neste sentido, para o autor, o sistema bipolar é muito mais estável que o multipolar. Com este aspecto, o seguinte trecho da obra de Waltz, pode traduzir a escalada de poder entre Estados:

Se cada Estado, sendo estável, lutasse apenas pela segurança e não cobiçasse seus vizinhos, todos os Estados, iriam não obstante, permanecer inseguros; porque os meios que garantem a segurança de um Estado são, pelo fato de existirem, os meios pelos quais os outros Estados são ameaçados. (WALTZ, 1979, p. 93).

Observa-se, portanto que não há como existir uma estabilidade, no sentido da negação de poder, já que os Estados estão em constante procura da expansão deste, ou seja, a anarquia é necessária e coopera neste sentido para equilibrar o sistema, já que a ação de competição entre Estados e o egoísmo dos mesmos conduz um estado racional do sistema.

Ao se trabalhar na perspectiva de Waltz, a ausência de um governo supranacional para aplicar regras de atuação para os Estados Ihe permite que estes apliquem suas vontades. Tomando como referência a citação, os Estados estão em constante escalada de capacidade de defesa, sendo dificultoso conceber em matéria de estudo uma nação que não busque ou o incremento da força e no caso de impossibilidade de aumento, a sua modernização. Neste sentido, a corrida pelo poder é constante e equilibra o sistema, impedindo o estabelecimento de uma potência única (WALTZ, 1979). 
No mesmo aspecto, a maximização do poder avança na mesma proporção das ambições individuais por um espaço na balança de poder. Os anseios expansionistas motivam essa maximização por parte do Estado, o qual, através desta, pontua a sua maneira de atuar no plano internacional, em si, determina o comportamento do Estado através da sua política exterior. Neste aspecto, a anarquia, a distribuição assimétrica do poder, a balança de poder, os anseios expansionistas dos Estados e o dilema da segurança criam uma condição na qual o embate bélico é iminente, e uma ameaça é algo presente (WALTZ, 1979).

Mearsheimer, autor de The Tragedy of Great Power Politics (2001), embora concorde com o sistema internacional construído por Waltz, critica que exista um equilíbrio de poder entre os Estados. Ele afirma que a estrutura do sistema internacional é o que as grandes potências fazem dela para, além de sobreviver, conquistar poder máximo de maneira ofensiva e assim descrê que as instituições sejam capazes de manter a paz por si só, pois o nacionalismo é posto acima da diplomacia interestatal. Portanto, as guerras são originadas pela constante competição de poder entre as hegemonias de maneira agressiva, pois raramente estão satisfeitas com suas posições na ordem.

Porém, Mearsheimer (2001) clama que conquistar a posição de hegemonia global é uma tarefa impossível de ser concluída, pois a expansão dos poderes estatais está restringida por um fator: os oceanos. Eles impedem que os Estados lancem seus projetos militares, econômicos e geográficos para o além-mar, de modo que possam controlá-los efetivamente. O seu território físico os constringe de manipular aquilo fora de seu alcance com eficácia e deixa brechas vulneráveis para que outros atores consigam controlá-las com maior imponência. Naturalmente, isso divide os poderes relativos do mundo. Assim, sem um hegemon para controlar tudo e a todos como um policial, os Estados estimulados pelos seus instintos de sobrevivência adotam três principais estratégias para expandir seus poderes e competirem por ele: hegemonia regional, prosperidade máxima e a superioridade nuclear.

O título de hegemonia regional é o que as partes mais buscam conquistar, porque hegemonias regionais possuem maiores probabilidades de garantir a sobrevivência no sistema. Para isso, como os países regionalmente não estão limitados pelos 
oceanos, procuram expandir ao máximo suas forças ideológicas, territoriais, econômicas e militares em relação aos países vizinhos, para alcançar a prosperidade. Esses, ao se sentirem intimidados, trabalharão também para aumentar os próprios poderes e competir pelo título (MEARSHEIMER, 2001).

A prosperidade máxima, além de estimular a competição regional, pontua para que a força econômica ganhe grande relevância, pois, através do aumento de capital e a disponibilidade de recursos, se possibilita a expansão do poder militar, o qual é a ferramenta principal da garantia de sobrevivência, do fortalecimento de defesas e de ataques e, por conseguinte, é a fonte de tensões por seu potencial destrutivo e opressivo, o que causa medo aos rivais (que ciclicamente fazem o mesmo) (MEARSHEIMER, 2001).

A maior fonte do seu potencial destrutivo é a superioridade nuclear, que as potências nucleares possuem, que devido ao seu potencial de destruição em massa, garante a capacidade dos atores de se dizimarem. As armas nucleares nada mais são que um apelo à sobrevivência estatal, pois podem ser utilizadas como última instância quando as opções de recursos para sobreviver se esgotam. Então, quando um Estado adquire poder nuclear, o usa de forma dissuasória para que os outros Estados realizem suas vontades ao se sentirem intimidados por sua força. Mas, quando mais de um país adquire este poder, o faz também em nome da posição de hegemonia e desafia este outro país no que o autor chama de destruição mutualmente certa (mutually assured destruction - MAD) (MEARSHEIMER, 2001).

Mearsheimer (2001) ainda conclui que, embora tenha um grande poder de dissuasão, a força nuclear não deixará o mundo contente com o uso de MAD para se regular. Assim, os países procurarão outros meios de ganhar superioridade sob seus rivais nucleares. Portanto, o ciberespaço pode ser comparado a isso. As teorias neorrealistas das Relações Internacionais tratam a tecnologia como um objeto neutro e desconsideram que ela pode ter qualquer influência relevante (HALPIN et. al, 2006). Partem da premissa de que a tecnologia é um instrumento influenciado apenas pela vontade do homem e não envolve consequências sociais (CARR, 2016). Essa 
perspectiva em que a tecnologia é tratada como algo estático é denominada instrumentalismo tecnológico (CARR, 2016).

Instrumentalismo é uma abordagem que observa a tecnologia como um meio de servir a um problema ou a uma necessidade humana. Inovação é sempre vista como positiva nessa perspectiva, se ela tiver "sucesso". Se a tecnologia funciona, ela é "boa". Para além disso, tecnologia é moralmente neutra e desconectada de suas consequências sociais. $O$ uso humano é que faz da tecnologia "boa" ou "má" - a tecnologia, por ela mesma, é avulsa de resultados morais[16] (CARR, 2016, p. 22, tradução nossa).

Nos últimos cinco séculos, porém, a evolução das tecnologias vem mudando e inchando a estrutura internacional, devido às interações cada vez mais rápidas e envolventes. Eles também vêm apresentando qualidades de semiautonomia, ou seja, os Estados não conseguem mais exercer o poder total sobre elas e são menos neutros para a sociedade, o que fragiliza parcialmente seu controle. Ao ignorar o papel chave das tecnologias nas relações internacionais, autores podem falhar em construir uma teoria de longo alcance ao instrumentalizá-las como apenas uma forma de ganhar poder (HALPIN et. al, 2006).

De acordo com a teoria determinística das tecnologias, a sociedade tem controle ou influência mínima. A autora Carr (2016), argumentam que as tecnologias têm sua própria força de governança, avançam de acordo com sua própria lógica (o que demonstra sua autonomia) e ainda impactam mais o desenvolvimento humano do que seus propósitos, porque diferentes povos, independentemente de suas culturas ou políticas, têm o mesmo, reação à mesma ferramenta (CARR, 2016 apud VIG, 1988 e BIMBER, 1990), ou seja, é a sociedade que se adapta às tecnologias e não a tecnologia às sociedades. Desta forma, é importante apontar o impacto das IAs na balança de poder, já que, diante do exposto, os Estados estão em constante busca de maximizar sua capacidade de influenciar seus competidores, expandindo seu alcance. Assim, a próxima seção busca apontar o impacto na balança de poder. 


\section{IAS E SEU IMPACTO NA BALANÇA DE PODER}

A configuração de segurança passa por um processo de digitalização dos espaços, o equilíbrio entre os Estados em razão das armas nucleares ocasiona uma ideia de segurança, no entanto, com a ascensão do uso da tecnologia no teatro de operações, apresenta-se um novo desafio para os atores estatais, já que, enraizados na revolução do computador, esses avanços estão tornando as forças nucleares em todo o mundo muito mais vulneráveis do que antes (LIEBER; PRESS, 2017). Na sua obra "The New Era of Counterforce" os autores Lieber e Press abordam a fragilidade dos sistemas de armas nucleares e como a automação - digitalização - desses sistemas erode o potencial de sobrevivência dos arsenais nucleares dos Estados. Segundo Lieber e Press (2017), com os sistemas atuais, nada poderia impedir um agente como uma IA de acessar um sistema de lançamento de ICBM[17] e desativar seus mecanismos.

Até o momento, o uso publicamente divulgado de IA para fins ofensivos tem sido limitado a experimentos de pesquisadores de "White Hats"[18], que visam aumentar a segurança, encontrando vulnerabilidades e sugerindo soluções (BRUNDAGE et al., 2018). No entanto, o ritmo de progresso das IAs sugere a probabilidade de ataques cibernéticos aproveitando os recursos de Machine Learning em estado selvagem, e em breve, se é que ainda não o fizeram. De fato, alguns relatos populares de IA e cybers-segurança incluem reivindicações baseadas em evidência circunstancial de que a IA já está sendo usada em práticas ofensivas por adversários sofisticados e motivados (BRUNDAGE et al., 2018)

Segundo Brundage et al. (2018), muitos governos estão profundamente interessados na combinação de IA e cyber-segurança. Os sistemas de IA já estão sendo definidos para desempenhar um papel expandido na estratégia e operações militares dos EUA, nos próximos anos, a medida que os EUA coloca em prática sua visão de uma estratégia de "Terceiro Desvio", em que humanos e máquinas trabalham juntos para alcançar objetivos militares (BRUNDAGE et al., 2018). Ao mesmo tempo, os governos estão investindo na pesquisa fundamental para expandir o escopo das capacidades de Sistemas de IA. O Almirante Mike Rogers, Diretor da Agência de Segurança Nacional, disse: "Inteligência Artificial e o aprendizado de máquina - eu diria - é 
fundamental para o futuro da cyber-segurança [...] Não é o se, é apenas o quando mim" (BRUNDAGE et al., 2018, n.p.).

Tecnologias emergentes moldam a balança de poder, primariamente através de meios militares e econômicos (HOROWITZ, 2018). Tecnologias alteram a balança de poder influenciando as habilidades de um país travar e ganhar guerras, dando a eles superioridade armamentista sobre seus rivais e um aumento na gama tática disponível para suas forças, criando a vantagem necessária para se sobressair. Mas, segundo Horowitz (2018), a maior vantagem que tecnologias inovadoras podem proporcionar é na área econômica, afinal, países não podem manter superioridade militar a longo prazo sem uma base econômica estável, remetendo ao declínio do império Otomano e a China imperial (HOROWITZ, 2018), assim:

O caráter da guerra em um período pode ser definido como a forma dominante de lutar e vencer conflitos, dadas as tecnologias, organizações e políticas existentes. O caráter da guerra muda em relação às ferramentas que se tornam disponíveis e como elas influenciam as formas como os militares se organizam para lutar nas guerras. A mudança para a mobilização em massa na era napoleônica exemplifica um desenvolvimento não tecnológico que mudou o caráter da guerra. (HOROWITZ, 2018, p. 11, tradução nossa).[19]

Brundage et al. (2018) discorre sobre os riscos políticos associados ao uso de IA maliciosa já que a mesma permite mudanças na natureza da comunicação entre indivíduos, empresas e Estados, de modo que estejam cada vez mais mediados por sistemas automatizados que produzem e apresentam conteúdo (BRUNDAGE et al., 2018). A tecnologia da informação já está afetando as instituições políticas em uma miríade de formas - por exemplo, o papel da mídia social em eleições, protestos, e até mesmo política externa. De acordo com Brundage et al. (2018), O uso crescente de IA pode fazer tendências existentes mais extremas e permitirem novos tipos de políticas dinâmica, é preocupante que os recursos da IA sejam particularmente adequados para minar o discurso público através da produção em larga escala de conteúdo falso, se tornando mais persuasivo e, possivelmente, causando o fortalecimento da mão de regimes autoritários. 
Al causará mudanças no cenário de segurança política, pois a corrida armamentista entre a produção e a detecção de informações enganosos evolui e os Estados buscam formas inovadoras de aproveitamento das IAs para manter sua soberania. Não está claro quais serão as implicações de longo prazo de tais usos maliciosos de IA, e esses casos discretos de mau uso de forma mais ampla apenas arranham a superfície das implicações políticas da IA . No entanto, esperamos que entender o panorama de ameaças encorajará medidas mais vigorosas de prevenção e mitigação. (BRUNDAGE et al., 2018, p. 45, tradução nossa).[20]

Os Estados que são capazes de controlar o poder da IA estão preparados para dominar a política, economia e diplomacia mundiais, além disso, hoje, os Estados têm menos probabilidade de vencer batalhas sem o uso de IA (ACHARYA, 2019). Não é difícil de imaginar que uma ferramenta tão útil e com um imenso potencial como as IAs se tornariam objetivo almejado pelas grandes potenciais globais. Segundo Horowitz (2018), os investimentos globais em Inteligências Artificiais para propósitos de segurança nacional e desenvolvimento econômico são descritos como uma corrida armamentista nos relatórios de segurança nacional dos países competidores. Sayler (2020) aborda a relação competitiva internacional de forma que, à medida que as aplicações militares para IA crescem em escala e complexidade, muitos na comunidade da defesa estão se tornando cada vez mais preocupados com a competição internacional. Hoje os Estados Unidos, China e Rússia possuem a vanguarda de desenvolvimento tecnológico na área (SAYLER, 2020).

De acordo com Horowitz (2018), a China publicou em 2017 no seu plano de estratégia nacional de IAs que as Inteligências Artificiais eram "uma grande oportunidade estratégica" e se propôs a uma estratégia para liderar as pesquisas tecnológicas na área. O autor continua explanando sobre a participação russa, inferindo que a Rússia está investindo principalmente no domínio militar, relatórios apontam que o exército russo está desenvolvendo veículos autônomos para guardar suas bases de misseis balísticos e um submarino autônomo que poderia portar armas nucleares, já na área robótica a Rússia está implantando em zonas de combate tanques remotamente pilotados, como o Uran-9 e Vehar. 
Segundo Horowitz (2018), além das potencias EUA, Rússia e China, outros países também estão interessados em implementar essas tecnologias nos seus interesses de segurança nacional. A natureza da tecnologia de IA possibilita e facilita que outros Estados se tornem aptos a aplicar essas tecnologias nos seus setores nacionais, nesse âmbito, as IAs podem equalizar a balança de poder em favor dos demais Estados, agindo como um multiplicador de forças. Outro grande problema que aparecera derivada da criação desta ferramenta, será o aumento das ameaças provindas de atores não-estatais, como grupos terroristas e cyber-terroristas.

Como elaborado nas seções prévias, as maiores vantagens que inteligências artificiais podem propiciar aos seus usuários são sua capacidade de processar uma quantidade gargantua de dados em uma margem de tempo mínima. Além de sua clara aplicabilidade nos setores de segurança, cyber-segurança e no setor econômico empenhá-la em conjunto aos tomadores de decisão da autoridade civil pode se provar extremamente beneficial. De acordo com Acharya (2019), a IA pode desempenhar um papel preditivo na geopolítica, prevendo resultados eleitorais, desempenho econômico e tomada de decisões de política externa.

Não é impossível para os estados projetarem máquinas de IA altamente qualificadas e designar diplomatas de IA, ministros de relações exteriores e pessoal militar de IA para diplomacia e defesa algum dia. Esses diplomatas inteligentes representarão nações e negociando em nome de Estados. Diplomatas de IA desempenhariam um papel significativo na tomada de decisões de política externa porque agem racionalmente e podem pensar vários passos à frente dos humanos. (ACHARYA, 2019, não paginado, tradução nossa).[21]

Ainda de acordo com Acharya (2019), essa tecnologia pode fazer suposições inteligentes sobre o melhor curso de ação futuro possível. As máquinas de IA são programadas com base em algoritmos de otimização e verificação que ajudam a perceber melhor uma sequência de informações em torno da esfera política. Os sistemas de IA podem prever eventos futuros, com base em eventos similares prévios, que possam oferecer oportunidades para os formuladores de política externa preverem as posições e táticas de seus oponentes, e até ajudar a modelar negociações complexas em assuntos internacionais (ACHARYA, 2019). 
Como elabora Bjola (2020), um sistema de IAG[22] aplicado em qualquer setor de um Estado, pode maximizar sua eficiência a um nível sobre-humano, ampliando suas capacidades, reduzindo o custo operacional, acrescentando mais possibilidades a seu leque de ações e aumentando o alcance e efetividade de suas políticas. No que discerne o uso de IAGs na assistência dos tomadores de decisão Bjola (2020) aponta, que há quatro principais áreas de ação da IA como assistente para tarefas especificas, crítico para revisão de trabalhos já concluído, uma segunda opinião e consultor. Elaborando nas palavras do autor, a máquina pode agir como um assistente invocado pelo tomador de decisão para realizar uma tarefa específica como parte de um exercício mais amplo. Quanto crítico revisando trabalhos já concluídos e comentando aspectos como precisão, consistência e completude. Quanto segunda opinião, executar a tarefa para que seus resultados possam ser comparados com os do tomador de decisão, bem como consultor, oferecendo conselhos ou opiniões com base nas informações fornecidas. Bjola (2020, p. 14) ilustra:

Um sistema de IA diplomática com conhecimento declarativo (KnowWhat) seria capaz de coletar, agregar e armazenar informações públicas sobre as posições das distintas partes na negociação. Uma com conhecimento processual (know-how) seria capaz de recuperar informações relevantes com base em certos critérios de entrada para auxiliar os negociadores em seu trabalho. Um sistema de IA com conhecimento condicional (Know-When) seria capaz de entender as condições para o uso de conhecimentos declarativos e procedimentais, como quando o sistema pode reconhecer o potencial de um avanço nas negociações e fornecer informações relevantes para que dê certo. Finalmente, um sistema de IA com conhecimento teleológico (KnowWhy) seria capaz de entender o propósito, intenção ou razão de usar conhecimento declarativo, procedimental ou condicional, quando as negociações servem para pôr fim a um conflito, mitigar as alterações climáticas ou para melhorar as condições para governança financeira.

Em suma, de uma perspectiva conceitual, os sistemas de IA são suficientemente desenvolvidos para fornecer suporte decisório de uma variedade de posições (assistente, crítico, segunda opinião, consultor) (BJOLA, 2020). Sua eficácia varia, no entanto, com a natureza da decisão (decisões estruturadas sendo mais passíveis de modelagem de IA) e o grau de interatividade entre o tomador de decisão e a máquina 
(conhecimento incorporado que sustenta a colaboração deve ser capaz de facilitar um melhor envolvimento do que o conhecimento que estimula a competição).

O impulso para aproveitar o poder da IA na tomada de decisões de política externa é informado por considerações sobre como gerenciar adequadamente as demandas de políticas e prioridades institucionais. Os autores Mintz e DeRouen (2010) apontam, o ambiente de tomada de decisão impõe várias restrições à capacidade dos formuladores de política externa para comparar, avaliar e buscar cursos de ação preferidos. O tempo é indiscutivelmente um fator crítico. Ser capaz de reagir rapidamente, especialmente no meio de uma crise, geralmente incentiva os tomadores de decisão a intencionalmente condensar o conjunto de alternativas, confiar mais em atalhos cognitivos e buscar uma solução satisfatória em vez de uma solução ótima (BJOLA, 2020).

Seguindo pela lógica apresentada acima, tornar-se adepto da arena cibernética nivela o campo de jogo - dando a países como o Irã e a Coreia do Norte a capacidade de bater de frente com a China, a Rússia e os EUA no espaço cibernético.

A busca pela supremacia da IA está proporcionando uma vantagem competitiva crescente no mercado internacional negócios para algumas nações menores, de outra forma menos competitivas, aumentando sua capacidade de assegurar acordos preferenciais de comércio e investimento com outros países, elevando seu perfil global e permitindoIhes progredir em áreas anteriormente inimagináveis de comércio internacional, investimento e diplomacia. (WAGNER; FURST, 2018, não paginado, tradução nossa).[23]

Wagner e Furst (2018) elaboram dizendo que, a maneira como a IA é implantada por governos pode ter sérias consequências nas relações internacionais, especialmente se um determinado governo tiver recursos incomuns na área de IA. O acesso e o uso de armas autônomas, podem alterar potencialmente o equilíbrio global de poder. Desafios para o equilíbrio de poder de uma região podem ocorrer à medida que alguns Estados se movem para alavancar a tecnologia de IA que facilitará reverter desvantagens militares históricas vis-à-vis seus vizinhos (WAGNER; FURST, 2018). No entanto, há muito pouco entendimento dentro dos Estados sobre como essas tecnologias funcionam e quais opções estão disponíveis para combatê-las. 
De acordo com Wagner e Furst (2018), Os governos começaram a planejar e investir para o futuro da IA, mas nenhum ainda desenvolveu e articulou linhas vermelhas acionáveis sobre como as tecnologias de IA podem ser usadas de acordo com as normas do direito internacional existente ou não. Com essa afirmação podemos fazer uma conexão ao teórico neorrealista John J. Mearsheimer onde no seu livro The Tragedy of Great Power Politics, aponta que, em meio da anarquia do sistema, todos os Estados almejam a sobrevivência, sendo atores racionais eles sempre buscam ter alguma vantagem sobre seus competidores (MEARSHEIMER, 2001).

Seguindo a lógica de Mearsheimer, o equilíbrio entre as potências entra em estresse estrutural vista essas ferramentas abaladoras, o autor Mearsheimer (2001) afirma que, é virtualmente impossível que um Estado consiga se tornar uma potência hegemônica, pois a principal dificuldade seria sua conseguir projetar seu poder na regia de seus rivais. Contudo, examinando todas as evidências dos potenciais das IAs e IAGs, do quão transformativas essas tecnologias de fato podem se provar, é possível imaginar uma potência hegemônica surgindo, um Estado similar ao Império Britânico no seu auge. Que por meio da primeira revolução industrial, que lhe forneceu a exclusiva vantagem das máquinas a vapor, conseguiu projetar seu poder através do mundo. Obviamente havia outras hegemonias regionais já estabelecidas, mas todas foram, de uma forma ou de outra, afetadas pelo expansionismo inglês.

\section{CONSIDERAÇÕES FINAIS}

As Inteligências Artificias se provam cada vez mais, uma das tecnologias que mais poderão afetar o progresso da humanidade a partir de meado dos séculos XXI e XXII, tal como o motor a vapor, a penicilina, a pólvora e a internet. Por consequência de serem ferramentas de automação e aceleração do processo de raciocínio, elas alterarão profundamente a forma como operamos, como sociedades e civilização, moldando novos métodos de interação entre os atores no cenário internacional. Diferente de outras ferramentas de poder, como as armas nucleares e porta-aviões de classe nuclear, que para sua manufatura necessitam de equipes altamente especializadas, materiais extremamente raros e altamente ilegais, um sistema de IA específico pode ser desenvolvido em uma garagem, em um computador gamer, por 
um único, ou mais indivíduos, bem motivados, que tenham uma graduação em sistemas da computação.

Em luz deste fato e dos que foram apresentados durante a pesquisa, pode-se começar a compreender o quão abaladora essa nova tecnologia pode se tornar, se for empregada de forma otimizada a auxiliar a operação dos sistemas estatais. A facilidade de replicação dessa ferramenta, sua natureza multiplicadora de forças e sua aptidão para maximizar os processos designados, trazem para a mesa de debate a questão de atores não estatais com más intenções, que podem utilizar dela para multiplicar geometricamente seu poder de ação. Além desses atores, Estados que não possuem a capacidade de projetar seu poder na sua região, ou até através do globo, terão suas habilidades potencializadas. Antes das possibilidades das IAs, tínhamos uma clara distinção de poder nacional, seja ele econômico ou bélico. Com seu surgimento é possível nivelar o campo internacional, trazendo calor as novas e velhas rivalidades.

Como a teoria neorrealista ofensiva de Mearsheimer defende, a ordem internacional é aquilo que as hegemonias fazem dela para se fortalecerem e, enquanto as potências tradicionais tentam manter suas posições de poder através da promoção de seus próprios meios tradicionais de poder, as reformistas tentam derrubá-las para ascenderem de posição. Portanto torna-se clara a competição entre China, Rússia e EUA, onde a China e Rússia são potências reformistas que tentam estender sua zona de influência através de laços comerciais, no caso da China, e projeção de poder militar, no caso da Rússia, os EUA, a tradicional, tenta conter esses avanços, reprimindo essas inovações e reafirmando os fatores que os fizeram superpotência. Esse padrão acaba se traduzindo para a corrida armamentista das IAs, onde Rússia busca a implementação de sistemas de armas autônomas, China com avanços na cyber-segurança e aplicações econômicas e EUA dependendo dos Think-Tanks Privados para sua supremacia. Porém, as teorias não abordam como a dominância sobre uma determinada tecnologia pode fazer com que os resultados sejam, de algum modo, decisivos, e nem como irão influenciar as relações internacionais. 
O fato das IAs, aplicadas como ferramentas geopolíticas, serem tão abstratas, tal como parte de um filme de ficção científica, de categoria $B$, vem do problema de que elas estão apenas na sua infância, o que torna difícil ponderarmos sobre suas capacidades máximas e ate onde podem chegar com suas habilidades. Mas devemos tratá-la como outras ferramentas que tinham o potencial de mudar as estruturas de poder, como a pólvora, que em sua infância era utilizada na China antiga como um instrumento de efeito moral, e hoje, é um instrumento essencial na arma mais destrutiva na história do universo. A Inteligência Artificial, seja ela utilizada como uma ferramenta extremamente útil para todas civilização humana, que talvez nos carregue para uma nova era dourada, ou a razão da nossa extinção como civilização.

Dessa forma, até no momento, é possível afirmar que as Inteligências Artificiais causarão sim um impacto na balança de poder e possivelmente reestruturando as atuais definições de segurança do Estado-Nação. Contudo é impossível prever quais serão estes impactos, pois a tecnologia não está completamente desenvolvida para ser aplicada de forma generalizada. Devido às restrições de espaço, tempo e a falta de informações sobre o assunto abordado, é recomendada uma pesquisa futura mais aprofundada no assunto, onde surgirão inovações tecnológicas e uma análise mais precisa a ser feita. No decorrer deste artigo descobriu-se mais do que era esperado. Os Estados não estão preparados para a chegada desta nova tecnologia, talvez porque ainda se imagina como algo futurístico, mas sem sombra de dúvidas, seu impacto será sentido, não apenas na relação entre os Estados e na forma como interagem, mas sim na vida de cada indivíduo da raça humana. O otimista pensa que este é o melhor de todos os mundos possíveis. O pessimista receia que isso seja verdade.

\section{REFERÊNCIAS}

ACHARYA, G. P. The Impacts of Al in International Relations. The Daily Star, [S. I.], $\quad$ p. $\quad$., $\quad 21 \quad$ Jul. $2019 . \quad$ Disponível em: $<$ https://www.thedailystar.net/opinion/perspective/news/the-impact-ai-internationalrelations-1774360>. Acesso em: 30 out. 2020. 
ALAN TURING, Biography. Engineering and Technology History Wiki, 2016. Disponivel em: $<$ https://ethw.org/Alan_Turing?gclid=CjwKCAiAn7LBRBbEiwAl9UtkFwl40QY-

_SaGhZ4GPMuNN2c0Umy2eGMQ9m9NHbncvqKK_J_LuF_AhoCZbsQAvD_BwE>. Acesso em: 5, dezembro de 2020.

ANDERSSON, Jenny. The Future of the World: Futurology, Futurists, and the Struggle for the Post-Cold War Imagination. Nova lorque: Oxford University Press. 2018.

BRUNDAGE, Miles et al. The Malicious Use of Artificial Intelligence: Forecasting, Prevention, and Mitigation. 2018. Disponível em: <https://img1.wsimg.com/blobby/go/3d82daa4-97fe-4096-9c6b376b92c619de/downloads/1c6q2kc4v_50335.pdf>. Acesso em: 31 out. 2020.

BJOLA, Corneliu. Diplomacy in the Age of Artificial Inteligence. Abu Dhabi, United Arab Emirates: Emirates Diplomatic Academy, 2020. Disponível em: <https://static1.squarespace.com/static/52c8df77e4b0d4d2bd039977/t/5e46b92b53c 62466ee47319c/1581693242795/EDA+Working+Paper_Artificial+Intelligence_EN+C opy.pdf. Acesso em: 2 nov. 2020>.

CAMPBELL, Murray; HOANE, A. Joseph; HSU, Feng-Hsiung. Deep Blue. Artificial Inteligence, ed. 134, p. 57-83, 2002. Disponível em: <https://reader.elsevier.com/reader/sd/pii/S0004370201001291?token=293DA9CC28 62EB3811A125E1ED88EFE0AC6410A14B1A376F82A1492E952A495DAE632ACF B595F9F9D03C5F22DBA3D143>. Acesso em: 17 set. 2020.

CARBONELL, Jaime G.; MICHALSKI, Ryszard S.; MITCHELL, Tom M. Machine Learning: A Historical and Methodological Analysis. Al Magazine, Englewood Cliffs, NJ: Prentice Hall, v. 4, n. 3, ed. 134, p. 69-78, 1972. Disponível em: $<$ https://www.researchgate.net/publication/2587421_Machine_Learning_A_Historical _And_Methodological_Analysis>. Acesso em: 17 set. 2020. 
CARR, Madeline. US Power and the Internet in International Relations: The Irony of the Information Age. Nova lorque: Palgrave Macmillan. 2016.

FREEDBERG, Sydney J. Al \& Robots Crush Foes in Army Wargame. Breaking Defense, 2019. Disponível em: <https://breakingdefense.com/2019/12/ai-robotscrush-foes-in-army-wargame/>. Acesso: 15, maio de 2020.

GAMBHIR, Mahak; GUPTA, Vishal. Recent Automatic Text Summarization Techniques: A Survey. Artificial Intelligence Review, [s. I.], v. 47, ed. 1, p. 1-66, 1 jan. 2017. Disponível em: <http://www.xuebalib.com/cloud/literature4njuzpxm3kZS.html>. Acesso em: 17 set. 2020.

HALPIN, Edward et. al. Cyberwar, Netwar and the Revolution in Military Affairs. Nova lorque: Palgrave Macmillan, 2006.

HE, Kaiming et al. Delving Deep into Rectifiers: Surpassing Human-Level Performance on ImageNet Classification. In: 2015 IEEE INTERNATIONAL CONFERENCE ON COMPUTER VISION, 2015, N/A. Delving Deep into Rectifiers: Surpassing Human-Level Performance on ImageNet Classification [...]. 2015. Disponível em: <https://sci-hub.do/https://doi.org/10.1109//CCV.2015.123>. Acesso em: 20 set. 2020.

HIGH-LEVEL EXPERT GROUP ON ARTIFICIAL INTELIGENCE (Europeian Comission). A Definition of Al: Main Capabilities and Scientific Disciplines. Bruxelas: $2019.290 \mathrm{p}$ Disponível em: https://ec.europa.eu/newsroom/dae/document.cfm?doc_id=56341>. Acesso em: 17 set. 2020.

HOROWITZ, Michael C. Artificial Intelligence, International Competition, and the Balance of Power. Texas National Security Review, [S. I.], v. 1, n. 3, p. 37-57, 1 maio 2018. Disponível em: <https://tnsr.org/2018/05/artificial-intelligence-internationalcompetition-and-the-balance-of-power/>. Acesso em: 13 out. 2020. 
KIM, Yoochul; LEE, Minhyung. Humans Are Still Better Than Al at StarCraft-for Now. MIT Technology Review, 1 Nov. 2017. Disponível em: <https://www.technologyreview.com/2017/11/01/241763/humans-are-still-better-thanai-at-starcraftfor-now/>. Acesso em: 16 set. 2020.

LANDGREBE, Jobst; SMITH, Barry. There is no Artificial General Inteligence. 2019. Disponível em: <https://arxiv.org/pdf/1906.05833.pdf>. Acesso em: 16 set. 2020.

LE, Quoc V.; SCHUSTER, Mike. A Neural Network for Machine Translation, at Production Scale. Google Al Blog, 27 jul. 2016. Disponível em: <https://ai.googleblog.com/2016/09/a-neural-network-for-machine.html>. Acesso em: 8 set. 2020.

LEVINE, Sergey et al. Learning Hand-Eye Coordination for Robotic Grasping with Deep Learning and Large-Scale Data Collection. International Journal of Robotics Research, Mountain View, CA, v. 37, n. 4-5, p. 421-436, 1 abr. 2018. DOI https://doi.org/10.1177/0278364917710318. Disponível em: <https://journals.sagepub.com/doi/full/10.1177/0278364917710318>. Acesso em: 14 set. 2020.

LIEBER, Keir A.; PRESS, Daryl G. The New Era of Counterforce: Technological Change and the future of Nuclear Deterrence. International Security, Vol. 41, No. 4 (Spring 2017), pp. 9-49, Disponível em: < https://www.belfercenter.org/sites/default/files/files/publication/isec_a_00273_LieberP ress.pdf>. Acesso em: 30 out. 2020.

LIETO, Antonio; CHELLA, Antonio; FRIXIONE, Marcello. Conceptual Spaces for Cognitive Architectures: A Lingua Franca for Different Levels of Representation. [S. l.: s. $\quad$ n.], $2017 . \quad 16 \quad$ p. $\quad$ Disponível em: <https://www.researchgate.net/publication/307917176_Conceptual_Spaces_for_Cog nitive_Architectures_A_Lingua_Franca_for_Different_Levels_of_Representation>. Acesso em: 17 set. 2020. 
NILSSON, NIls J. The Quest for Artificial Inteligence: A History of Ideas and Achievements. Cambridge: Cambridge University Press, [s. I.], 2010. DOI https:// doi.org/10.1017/CBO9780511819346.

Disponível em: <https://doi.org/10.1017/CBO9780511819346>. Acesso em: 16 set. 2020.

NGUYEN, Anh; YOSINSKI, Jason; CLUNE, Jeff. Deep Neural Networks are Easily Fooled: High Confidence Predictions for Unrecognizable Images. 2015. 10 p. Disponível em: $<$ https://www.researchgate.net/publication/307560165_Deep_neural_networks_are_ easily_fooled_High_confidence_predictions_for_unrecognizable_images $>$. Acesso em: 17 set. 2020.

MCCARTHY, John. WHAT IS ARTIFICIAL INTELLIGENCE?. Stanford University, 12 nov. 2007. Disponível em: <http://jmc.stanford.edu/articles/whatisai/whatisai.pdf>. Acesso em: 20 out. 2020.

MEARSHEIMER, John. The Tragedy of Great Power Politics. Nova lorque: W.W. Norton \& Company, 2001.

METZ, Cade. In Two Moves, AlphaGo and Lee Sedol Redefined the Future. WIRED, [S. I.], 16 mar. 2016a. Disponível em: <https://www.wired.com/2016/03/two-moves-alphago-lee-sedol-redefined-future/>. Acesso em: 10 set. 2020.

METZ, Cade. In a Huge Breakthrough, Google's Al Beats a Top Player at the Game of Go. WIRED, [s. I.], 27 jan. 2016b. <Disponível em: https://www.wired.com/2016/01/in-a-huge-breakthrough-googles-ai-beats-a-topplayer-at-the-game-of-go/>. Acesso em: 10 set. 2020.

MINTZ, Alex; DEROUEN, Karl. Understanding Foreign Policy: Decision Making. Cambridge University Press, Cambridge, MA, p. 25-30, 2010. Disponível em: <https://doi.org/10.1017/CBO9780511757761>. Acesso em: 1 nov. 2020. 
MORAVEC, Hans. Mind Children: The future of robot and human intelligence. Cambridge Mass: Harvard University Press, Cambridge, MA, p. 15, 1988.

MORGAN, Forrest E. et al. Military Applications of Artificial Intelligence: Ethical Concerns in an Uncertain World. Santa Monica, California: RAND Corporation, 2020. Disponível em: <https://www.rand.org/content/dam/rand/pubs/research_reports/RR3100/RR31391/RAND_RR3139-1.pdf>. Acesso em: 28 set. 2020.

RILEY, Tonya. Artificial Intelligence Goes Deep to Beat Humans at Poker. Science, [s. $\quad$ l.], $\quad 3 \quad$ mar. 2017.2 Disponível em: <https://www.sciencemag.org/news/2017/03/artificial-intelligence-goes-deep-beathumans-poker>. Acesso em: 13 set. 2020.

RUSSIA: 'Whoever leads in Al will rule the world - Putin at National Knowledge Day. Ruptly, $2017 . \quad$ Disponível em: <https://www.youtube.com/watch?v=xPuAzc3Y_64>. Acesso em: 3 nov. 2020.

SAYLER, Kelley M. Artificial Intelligence and National Security. Congressional Research Service, [S. I.], n. 8, p. 20-26, 26 ago. 2020. Disponível em: <https://fas.org/sgp/crs/natsec/R45178.pdf>. Acesso em: 29 out. 2020.

SIGNIFICADOS. Significado de Machine Learnig. Disponivel em: <https://www.significados.com.br/machine-learning/>. Acesso em: 17 set. 2020.

VILAR LOPES, Gills; ALMEIDA MEDEIROS, Marcelo de. CiberRI ou introdução aos estudos sistemáticos sobre o ciberespaço no tripé ensino-pesquisa-extensão de Relações Internacionais. Revista Meridiano 47 do Instituto Brasileiro de Relações Internacionais - IBRI, v. 19, 2018.

WAGNER, Daniel; FURST, Keith. Al and the International Relations of the Future. INTERNATIONAL POLICY DIGEST, 12 ago. 2018. Disponível em: $<$ https://intpolicydigest.org/2018/08/12/ai-and-the-international-relations-of-thefuture/>. Acesso em: 1 nov. 2020. 
WALTZ, Kenneth. Man, the State, and War. Nova lorque: Columbia University Press. 1959.

WALTZ, Kenneth. Theory of International Politics. Boston: Addison-Wesley Pub. Co. 1979.

\section{APÊNDICE - REFERÊNCIAS DE NOTA DE RODAPÉ}

3. "Relações Internacionais Cibernéticas (CiberRI) é um subcampo internacionalista que trata de temas afeitos ao ciberespaço, bem como de seus impactos nas relações internacionais e vice-versa. Assim, advoga-se a favor de a comunidade epistêmica adentrar, sistematicamente, nas discussões sobre o ciberespaço, as quais aparentam ser distantes dos estudos tradicionais de Relações Internacionais (RI)" (VILLAR LOPES; ALMEIDA MEDEIROS, 2018, paginação irregular).

4. Futurologia parte da ótica que o futuro, conforme as tendências, não resultará em algo positivo para a sociedade. Dessa forma, possui um caráter um tanto pessimista sobre os acontecimentos. É considerado de certa perspectiva a antítese da utopia, ou seja, uma distopia (ANDERSSON, 2018).

5. IAGs: Inteligencias Artificiais Generalizadas.

6. Teste de Turing foi criado por Alan Turing, que foi um matemático e cientista inglês amplamente considerado o fundador da disciplina de ciência da computação. A máquina de Turing, um conceito abstrato que detalha muitas das ideias fundamentais da ciência da computação, é um dos marcos mais importantes na história da computação. (ALAN TURING Biography, 2016).

7. Tradução nossa: "Artificial intelligence (Al) systems are software systems (and possibly also hardware) designed by humans that, meeting a complex objective, act in the physical or digital dimension, perceiving their environment through data acquisition, interpreting structured data or unstructured data collected, thinking about knowledge or processing information, derived from this data and deciding the best action (s) to achieve the determined objective." 
8. "Machine Learning é uma área da ciência da computação que significa "aprendizado da máquina". Faz parte do conceito de inteligência artificial, que estuda meios para que máquinas posam fazer tarefas que seriam executadas por pessoas" (SIGNIFICADOS, 2020).

9. Tradução nossa: "Dislodge a defense infantry company, about 120 soldiers, with a single platoon of just 40 attackers on foot. This is a task that would normally be assigned to a battalion of more than 600. In other words, instead of the minimum 3:1 superiority in numbers that military tradition requires for a successful attack, Belanger's simulated strength was in lowest number 1: 3."

\section{DARPA: Defense Advanced Research Projects Agency}

11. Tradução nossa: "In terms of the complexity of the board positions and the number of possible moves, Go is a much more difficult game than chess, so Go enthusiasts, and even Al researchers, thought it would be another decade before computers reached performance superhuman in that game".

12. O OODA Loop é o ciclo observar-orientar-decidir-agir, desenvolvido pelo estrategista militar e Coronel da Força Aérea dos Estados Unidos, John Boyd.

13. Tradução nossa: "With the idea that if it were possible to operate through the OODA Loop faster than their opponents, then they will be unable to take the necessary counter-actions to defend against their own attacks or to generate their own offensive options fast enough to overcome the counter-actions".

14. Tradução nossa: "The term Big Data is generally used to describe data that is too large to be stored in the computer's memory, is generated too quickly to be managed by a single computer, or many different forms or formats. Because of these three problems, it can be challenging for humans to make sense of the information contained in the data, but machines and Al tend to perform better the more data is made available to them. the large volume of information collected by various sensors is more than a human or team of humans can analyze" 
15. Tradução nossa: "it is no longer necessary to simply use static tags to identify malware, because attackers have found ways to generate malware with fewer of these tags. In response, how antivirus companies analyzed their large malware behavior data sets to create Al that can watch software on a system and signal actions that are identified as suspicious.".

16. Tradução nossa: "Instrumentalism is an approach which regards technology as a means to address a human-defined problem or need. Innovation is always regarded in this approach as positive if it is 'successful'. If technology works, it is 'good'. Beyond this, technology is morally neutral and disconnected from its social consequences. Human use makes technology 'good' or 'evil' - the technology itself is detached from moral outcomes."

\section{ICBM: Intercontinental Ballistic Missile.}

18.Um hacker de chapéu branco é um indivíduo que usa habilidades de hacking para identificar vulnerabilidades de segurança em hardware, software ou redes. No entanto, ao contrário dos hackers de chapéu preto, os hackers de chapéu branco respeitam o estado de direito no que se refere ao hacking.

19. Tradução nossa: "The character of war in a period can be defined as the dominant way of fighting and winning conflicts, given the existing technologies, organizations and policies. The character of war changes in relation to the tools that become available and how they influence the ways in which the military organizes itself to fight wars. The shift to mass mobilization in the Napoleonic era exemplifies a nontechnological development that changed the character of the war.".

20. Tradução nossa: "Al will cause changes in the political security scenario, as the arms race between the production and detection of misleading information evolves and States are looking for innovative ways to take advantage of Als to maintain their sovereignty. It is unclear what the long-term implications of such malicious uses of $A$ I will be, and these discrete cases of misuse more broadly only scratch the surface of 
Al's political implications. However, we hope that understanding the threat landscape will encourage more vigorous prevention and mitigation measures.".

21. Tradução nossa: "It is not impossible for states to design highly qualified Al machines and designate Al diplomats, foreign ministers and Al military personnel for diplomacy and defense someday. These smart diplomats will represent nations and negotiate on behalf of states. Al diplomats would play a significant role in making foreign policy decisions because they act rationally and can think several steps ahead of humans".

22. IAG: Inteligência Artificial Generalizada.

23. Tradução nossa: "The quest for Al supremacy is providing a growing competitive advantage in the international business market for some smaller, otherwise less competitive nations, increasing their ability to secure preferential trade and investment agreements with other countries, raising their global profile and enabling them make progress in previously unimaginable areas of international trade, investment and diplomacy.".

Enviado: Janeiro, 2021.

Aprovado: Janeiro, 2021. 